\title{
A prospective phase II study of chemoradiation followed by adjuvant chemotherapy for FIGO stage I-Illa (1988) uterine papillary serous carcinoma of the endometrium
}

\author{
Anuja Jhingran, M.D. ${ }^{a}{ }^{\star}$, Lois M. Ramondetta, M.D. ${ }^{b}$, Diane C. Bodurka, M.D. ${ }^{b}$, Brian M. \\ Slomovitz, M.D. ${ }^{d}$, Jubilee Brown, M.D. ${ }^{b}$, Lawrence B. Levy, M.S. ${ }^{c}$, Michael E. Garcia, R.N. ${ }^{b}$, \\ Patricia J. Eifel, M.D. ${ }^{a}$, Karen H. Lu, M.D. ${ }^{b}$, and Thomas W. Burke, M.D. ${ }^{b}$ \\ aDepartment of Radiation Oncology, The University of Texas MD Anderson Cancer Center, \\ Houston, TX 77030 \\ ${ }^{b}$ Department of Gynecologic Oncology and Reproductive Medicine, The University of Texas MD \\ Anderson Cancer Center, Houston, TX 77030 \\ 'Department of Biostatistics, The University of Texas MD Anderson Cancer Center, Houston, TX \\ 77030
}

dWomen's Cancer Center, Atlantic Health, Morristown Memorial Hospital, Morristown, NJ 07962

\begin{abstract}
Objective-To prospectively evaluate tumor control, survival, and toxic effects in patients with International Federation of Gynecology and Obstetrics (1988) stage I-IIIA papillary serous carcinoma of the endometrium treated with concurrent chemoradiation and adjuvant chemotherapy.
\end{abstract}

Methods-Thirty-two patients were enrolled from October 2001 through July 2009. Patients underwent full surgical disease staging and postoperative concurrent weekly paclitaxel $\left(50 \mathrm{mg} / \mathrm{m}^{2}\right)$ and pelvic RT to 45 Gy plus a vaginal cuff boost followed by 4 cycles of adjuvant paclitaxel (135 $\left.\mathrm{mg} / \mathrm{m}^{2}\right)$.

Results-Thirty patients (94\%) were evaluable (3 with stage IA disease, 11 IB, 3 IC, 1 IIB, and 12 IIIA). Eighteen patients (60\%) received all 5 planned courses of concurrent chemotherapy, 10 (33\%) received 4 courses, and 2 (7\%) received 3 courses. All 30 patients received RT; 27 (90\%) received the full dose, 2 received $43.2 \mathrm{~Gy}$, and 1 received 39.6 Gy owing to toxic effects. Twenty-

\footnotetext{
*To whom correspondence and reprint requests should be addressed: Department of Radiation Oncology, Unit 1202, The University of Texas MD Anderson Cancer Center, 1515 Holcombe Blvd., Houston, Texas 77030. Phone: (713) 563- 2347. FAX: (713) 792-3642, ajhingra@mdanderson.org.

Presented at the 41st Annual Society of Gynecologic Oncologists Meeting on Women's Cancer, March 14-17, 2010, San Francisco, California.

Conflict of Interest Statement: Diane C. Bodurka: Consultant: Genentech Otherwise there are no other financial conflicts of interest.

Publisher's Disclaimer: This is a PDF file of an unedited manuscript that has been accepted for publication. As a service to our customers we are providing this early version of the manuscript. The manuscript will undergo copyediting, typesetting, and review of the resulting proof before it is published in its final citable form. Please note that during the production process errors may be discovered which could affect the content, and all legal disclaimers that apply to the journal pertain.
} 
three patients (77\%) completed all 4 cycles of adjuvant paclitaxel, 3 (10\%) completed 3 cycles, 2 (7\%) completed 2 cycles, and 2 received no adjuvant therapy. Overall survival (OS), progressionfree survival (PFS), and local control rates for all patients were 93\%, 87\%, and 87\%, respectively, at 2 years and $85 \%, 83 \%$, and $87 \%$, respectively, at 5 years. Six patients developed (20\%) grade $3 / 4$ toxcities from the treatment. Four patients (13\%) had grade 3 or more severe bowel complications and two patients developed symptomatic pelvic fractures.

Conclusions-Treatment with concurrent paclitaxel and pelvic RT followed by 4 courses of systemic paclitaxel produced favorable results in patients with surgically staged I-III UPSC.

\section{Keywords}

Endometrial carcinoma; radiotherapy; papillary serous; chemotherapy; concurrent chemotherapy and radiation therapy

\section{Introduction}

Uterine papillary serous carcinoma (UPSC) is an uncommon but aggressive subtype of endometrial cancer. UPSC behaves more like ovarian than endometrial carcinoma in its propensity for early and intraperitoneal spread; prognosis even for patients with stage I disease is poor, with 5-year overall survival (OS) rates ranging from $45 \%$ to $78 \%$ [1-6]. Because of its propensity for early and intraperitoneal spread, UPSC is thought to require whole-abdominal or possibly systemic treatment for adequate coverage of potential disease sites; indeed, treatments evaluated for UPSC have included whole abdominopelvic irradiation (WAPI) and chemotherapy. Unfortunately, WAPI has been associated with poor disease-free survival (5-year disease-free survival rates of 40-50\%) and high recurrence rates even when applied in an adjuvant setting [4,7].

There are histologic and clinical similarities between UPSC and ovarian papillary serous carcinoma which might suggest that the two tumors have similar chemotherapeutic sensitivities and response rates. However, whereas the response rate of ovarian cancers to platinum-based therapy (without paclitaxel) is $60 \%$ to $80 \%$, the response rate of UPSC to platinum-based therapy has typically been less than $25 \%$ with a median duration of response of less than 1 year [8-10]. Higher response rates for UPSC have been reported with paclitaxel: a combined response rate of $89 \%$ was reported in patients with UPSC receiving either single-agent paclitaxel or paclitaxel and cisplatin as adjuvant therapy [11].

At The University of Texas MD Anderson Cancer Center, we previously completed a phase II trial evaluating whether paclitaxel improved local control (LC) and survival for patients with advanced or recurrent UPSC. In that trial, 13 patients had measurable disease, and among those 13 patients, 10 patients (77\%) had an objective response (4 complete clinical responses and 6 partial responses) and 3 patients (23\%) had stable disease [12]. Paclitaxel has been theorized to be an effective radiation sensitizer because it promotes microtubule assembly and results in cell cycle arrest at $\mathrm{G}_{2} / \mathrm{M}$, the most radiosensitive phase of the cell cycle $[13,14]$. Research indicates that paclitaxel induces cellular apoptosis independent of tumor p53 status and therefore may complement radiation therapy (RT) in inducing apoptosis [15]. Several phase I trials have demonstrated the efficacy and safety of weekly 
paclitaxel administered with RT. The weekly maximum tolerated dose of $50 \mathrm{mg} / \mathrm{m}^{2}$ has been associated with minimal and manageable toxic effects, including diarrhea, nausea, and neutropenia [16-18].

Because of the results of our previous phase II trial and the data suggesting synergy between paclitaxel and RT, we decided to proceed with a phase II trial to evaluate whether adding concurrent pelvic RT to paclitaxel for patients with UPSC would improve the results seen with paclitaxel alone.

\section{Methods}

This study was approved by the Institutional Review Board of MD Anderson, and informed consent was obtained from all patients. Patients were enrolled at MD Anderson in Houston or at its affiliate in Orlando, Florida. The planned treatment was surgery including hysterectomy and staging; postoperative concurrent chemotherapy and pelvic RT consisting of weekly paclitaxel $\left(50 \mathrm{mg} / \mathrm{m}^{2}\right)$ and 45 Gy to the pelvis and a vaginal cuff boost; and finally 4 cycles of paclitaxel at $135 \mathrm{mg} / \mathrm{m}^{2}$. The various treatments are described in detail below. The primary objective of the study was to evaluate the results of paclitaxel and pelvic RT for pelvis-confined UPSC in terms of both LC and OS.

All patients underwent a surgical staging procedure, defined as a total abdominal hysterectomy, bilateral salpingo-oophorectomy, pelvic cytologic evaluations, omental biopsy, and sampling of at least the pelvic lymph nodes. Disease stage was assigned according to the International Federation of Gynecology and Obstetrics (FIGO) surgical staging criteria set forth in 1988[19]. All histopathologic diagnoses were made by gynecologic pathologists at MD Anderson. The histologic tumor type (i.e., pure UPSC or mixed carcinoma involving serous and other cell types) was determined on the basis of uterine pathology. Patients who had any serous component in their pathologic specimens were considered eligible for this study. Patients with only clear cell in the specimen were not eligible for the protocol. Patients had to have non-measurable surgically staged FIGO 1988 stage I-IIIA disease, had to have a Zubrod performance status of 2 or less, and had to be enrolled in the study within 8 weeks after surgery.

\section{Radiation therapy}

Patients were treated with external-beam (EB) RT followed by high-dose-rate (HDR) brachytherapy with vaginal dome cylinders. All patients were to undergo EB RT delivered to the pelvis only, to a total dose of $45 \mathrm{~Gy}$ in 25 fractions over 5 weeks. Patients were treated once a day, 5 days a week, with daily fractions of $1.8 \mathrm{~Gy}$. A 4-field technique was used with anterior, posteriorand right and left lateral fields using 18-MV photon beams.

The superior border of all fields was placed at the L4-L5 interspace, and the inferior border was placed through the mid-pubis or $4 \mathrm{~cm}$ beyond the most distal vaginal disease. The lateral borders of the anterior and posterior fields were placed 1 to $1.5 \mathrm{~cm}$ lateral to the widest true pelvis diameter. The anterior border of the lateral fields was placed through the symphysis pubis and at least $1 \mathrm{~cm}$ anterior to the common iliac nodes at L4-L5, and the 
posterior border was placed at S2-S3. Intensity-modulated radiation therapy (IMRT) was not allowed.

The vaginal apex was treated after EB RT with HDR brachytherapy. A Delclos dome cylinder was used in all cases, and patients were to receive a total of $15 \mathrm{~Gy}$ in 3 fractions, all prescribed to the surface of the dome.

\section{Chemotherapy}

Paclitaxel $50 \mathrm{mg} / \mathrm{m}^{2}$ was administered via intravenous (IV) infusion over 1 hour in an outpatient setting on days $1,8,15,22$, and 29 during RT, to be followed by 4 additional courses of paclitaxel $135 \mathrm{mg} / \mathrm{m}^{2}$ IV over 3 hours every 21 days after the pelvic RT was completed. Dexamethasone $20 \mathrm{mg}$, diphenhydramine $50 \mathrm{mg}$, and cimetidine $300 \mathrm{mg}$ were given intravenously to all patients 30 minutes before administration of chemotherapy; patients who developed any allergic reactions were given dexamethasone $20 \mathrm{mg} 12$ hours and 6 hours before paclitaxel administration, and for such patients a desensitization regimen was used. Paclitaxel was administered via infusion control device (pump) using nonpolyvinylchloride tubing and connectors, and nothing else was infused through these lines. Dose modifications for hematologic, cardiac, and neurologic toxic effects were clearly outlined in the protocol. Paclitaxel was to be discontinued if the patient required more than 2 dose reductions. Patients were allowed to receive granulocyte colony-stimulating factor, but this was to be discontinued at least 24 hours before the next course of chemotherapy.

\section{Statistical methods}

This was an open-label, phase II study of IV paclitaxel given weekly with pelvic RT for 5 weeks followed by 4 courses of paclitaxel alone for patients with pelvis-confined UPSC. The purpose of the study was to determine the acute and late toxic effects of weekly paclitaxel concomitant with daily pelvic RT and to determine OS and time to local recurrence. The results were analyzed on an intent-to-treat basis. The primary endpoint of the study was overall survival and time to recurrence. The two-year recurrence-free rate was assumed to be $50 \%$ with surgery alone and the study was designed to demonstrate a $70 \%$ improvement due to the use of this treatment over surgery alone. Assuming exponential survival, an accrual time of 4.1 year was required to provide an $80 \%$ chance of detecting the improvement stated using a one-side test with significance level of 0.05 . The expected number of patients entered was 49 , however since there was such a slow accrual of only 32 patients in 8 years, it was decided to stop the study before reaching full accrual.

Log-rank tests were used to assess potential prognostic factors, including patient age, disease stage, positive cytologic findings on examination of peritoneal washing, ovarian/ fallopian tube involvement, and lymphovascular space invasion. OS, LC, and PFS were estimated on the basis of Kaplan-Meier curves. Cox regression analysis was performed to compare data from a historical study group [5] with data from the patients in the current study. SPSS software was used for all analyses. 


\section{Results}

Thirty-two patients were enrolled in the study from October 2001 through July 2009. Two patients were not evaluable for analysis, 1 who withdrew during pelvic RT and was lost to follow-up and a second one who was found to have pancreatic cancer during treatment, leaving 30 patients for analysis. The median age of the 30 evaluable patients was 63 years (range, 36- 77 years). The median follow-up time for all patients from the time of registration was 46 months (range, 6-111 months). Patient characteristics are shown in Table 1 .

\section{Treatment}

Twenty-seven patients (90\%) completed 25 of 25 planned radiation treatments to the pelvis, 2 patients $(6 \%)$ received 24 treatments, and 1 patient $(4 \%)$ received 21 treatments. All patients received 3 HDR vaginal cuff boost doses. Eighteen patients $(60 \%)$ received 5 of 5 planned courses of weekly paclitaxel during pelvic RT, 10 patients (33\%) received 4 courses, and 2 patients (7\%) received only 3 courses. Paclitaxel was withheld during RT because of diarrhea (3 patients), low absolute neutrophil count (3 patients), and other reasons, including fever, rectal bleeding, low potassium level, elevated liver function test findings, and stomatitis.

Twenty-three patients $(77 \%)$ received all 4 planned courses of paclitaxel $\left(135 \mathrm{mg} / \mathrm{m}^{2}\right)$ after chemoradiation, 3 patients (10\%) received 3 courses, and 2 patients (7\%) received 2 courses. Two patients (7\%) withdrew from the study after completing chemoradiation and therefore did not receive further chemotherapy.

\section{Acute toxic effects}

All toxic effects during treatment are listed in Tables 2 and 3. Most of the grade 3 or 4 hematologic toxic effects occurred during the systemic chemotherapy given after chemoradiation. All cases of fatigue and diarrhea occurred during chemoradiation. One patient received only 21 of 25 planned fractions of pelvic RT because of severe diarrhea, which resolved before post-chemoradiation systemic chemotherapy was begun. One patient with a history of myocardial infarction had another myocardial infarction during postchemoradiation systemic chemotherapy and therefore received only 2 of the planned 4 courses. A patient with grade 4 bone pain at the end of her treatment had grade 2 bone pain before the start of chemoradiation therapy; her treatment was stopped early because of a low absolute neutrophil count, after which she refused further treatment.

Five patients required growth cell stimulating factors (G-CSF) during their treatments. Three patients received Aranesp during systemic paclitaxel. One patient received neupogen injections during chemoradiation. This patient became neutropenic after week one and received her first dose; however after the $2^{\text {nd }}$ injection she was unable to take more due to bone pain. She received 2 more injections during week 3 of her chemoradiation therapy but was unable to take any more injections due to pain and then withdrew from the study following the completion of chemoradiation therapy and did not receive any systemic 
chemotherapy. The fifth patient received 2 injections of neupogen just prior to the $1^{\text {st }}$ dose of systemic paclitaxel but was unable to tolerate any more injections due to chest pain.

\section{Treatment results}

The OS, PFS, and LC rates at 2 years for the entire group were $93 \%, 87 \%$, and $87 \%$; the corresponding rates at 5 years were $85 \%, 83 \%$, and $87 \%$ (Fig. 1). For patients with stage I disease, the OS, PFS, and LC rates at 2 years were $100 \%, 94 \%$, and $94 \%$, and the corresponding rates at 5 years were $93 \%, 94 \%$, and $94 \%$. For patients with stage IIIA disease, the OS, PFS, and LC rates at 2 years were $83 \%, 75 \%$, and $75 \%$, and the corresponding rates at 5 years were $74 \%, 67 \%$, and $75 \%$. There was not a significant difference in OS or LC between patients with stage I and those with stage IIIA disease ( $\mathrm{p}=$ 0.42 and $p=0.14$, respectively); however, there was a significant difference in PFS between the 2 groups $(\mathrm{p}=0.025)$. Six patients experienced disease recurrence, and 4 of them died of the disease. The four patients that died of their disease developed diffuse peritoneal recurrences both in the abdomen and pelvis. The 1 patient who is still alive after recurrence at the time of this report underwent surgical resection of her recurrent disease and adjuvant chemotherapy. She recurred with a peritoneal nodule that was just outside the radiated field. A sixth patient developed recurrent disease in the inguinal nodes, received additional RT, and is alive without evidence of disease at the time of this report. Univariate analyses showed that stage IIIA disease correlated with reduced PFS $(p=0.02)$; no other factor examined correlated with PFS. Patient age, disease stage, positive cytologic findings on examination of peritoneal washing, ovarian/fallopian tube involvement, and lymphovascular space invasion were not correlated with OS or LC. Unfortunately, because the study was closed before complete accrual, it did not meet the primary endpoint threshold. With a sample size of 30 patients, the study had a power of $73 \%$ to yield statistically significance.

\section{Late toxic effects}

Among the 30 evaluable patients, 4 (13\%) experienced severe ( $\geq$ grade 3 ) bowel toxic effects: 3 had small bowel obstructions ( 2 requiring surgery and 1 treated conservatively), and 1 experienced chronic diarrhea that affected quality of life. One of these 4 patients also developed a sacral sarcoma, approximately 6 years after treatment. This patient declined treatment for the sarcoma and died of disease. Two patients developed symptomatic pelvic fractures.

\section{Discussion}

UPSC behaves more like ovarian than endometrial carcinoma in its propensity for early and intraperitoneal spread; prognosis even for patients with stage I disease is poor, with 5-year OS rates ranging from $45 \%$ to $78 \%$ [1-6]. In this phase II trial, we found that concurrent weekly paclitaxel with pelvic RT followed by 4 courses of systemic paclitaxel produced favorable 5-year rates of OS, PFS, and LC for women with stage I-IIIA UPSC (85\%, 83\%, and $87 \%$ for the entire group).

Our results for stage I disease compare favorably with those of other retrospective published results for stage I disease. For example, in a report by Kwon et al. [4] on 23 women with 
stage I UPSC treated with WAPI only (no chemotherapy), the 5-year survival rate was 78\%. In another retrospective review, Fader et al. [20] described 142 patients with surgical stage I UPSC, 33 of whom were treated with observation after surgery, 20 with RT alone, and 89 with chemotherapy with or without RT. The chemotherapy was primarily carboplatin and paclitaxel, and the RT was either vaginal cuff brachytherapy alone (32\% of patients) or pelvic and vaginal cuff brachytherapy (68\% of patients). The 5-year DFS rate for all patients in the study was $82 \%$ for those treated with chemotherapy with or without RT, $64 \%$ for those treated with RT alone, and $65 \%$ for those treated with observation alone.

Most other studies, including a previous study from our own institution, have reported results for patients with all stages of UPSC combined. Our results compare favorably with those of a 2003 retrospective review of 129 patients with UPSC treated at MD Anderson from 1989 through 2002 [5]. In that study, there were 65 patients with stage I-IIIA disease; they had been treated with observation (34 patients), preoperative brachytherapy (6 patients), vaginal cuff brachytherapy alone (6 patients), whole pelvic RT or WAPI (5 patients), or systemic chemotherapy (5 patients). Of these 65 patients, 51 had stage I disease and 14 had stage IIIA disease. Five of the patients with stage I disease (10\%) received chemotherapy. Comparison of all patients in the current study group with all patients in this historical series revealed improvements in OS and PFS for patients treated on our protocol ( $\mathrm{p}<0.001$ and $\mathrm{p}=0.0062$, respectively) (Figs. 2). Protocol-treated patients with stage I disease had significantly better OS and PFS than the patients from the historical series ( $\mathrm{p}=$ 0.0043 and $p=0.03$ ) (Fig. 3), and protocol-treated patients with stage IIIA disease had significantly better 5-year OS and PFS than patients in the historical series ( $\mathrm{p}=0.03$ and $\mathrm{p}=$ 0.04, respectively) (Fig. 4).

Our findings from the current phase II study are in agreement with findings from 2 recent retrospective studies suggesting that a combination of RT and chemotherapy may be beneficial in the treatment of patients with stage I-IV UPSC [21,22]. One study was done through the Rare Cancer Network [21]. This study included 129 patients with stage I-IV papillary serous carcinoma. Twenty-three patients received platinum-based chemotherapy, 52 patients received RT alone, and 28 received combined chemoradiation. For all patients, the 5-year DFS and OS rates were $42 \%$ and 54\%, respectively. In a multivariate analysis, adjuvant chemotherapy significantly improved DFS, and RT reduced the pelvic recurrence rate from $29 \%$ to $14 \%(\mathrm{p}=0.047)$. The other recent retrospective study of chemoradiation for UPSC was a study of 135 patients with stage I-IV UPSC treated with observation $(n=36)$, chemotherapy alone $(n=10)$, or RT with or without chemotherapy $(n=89)$ [22]. The authors found that the combination of RT and paclitaxel-based chemotherapy was associated with longer relapse-free survival and that paclitaxel-platinum chemotherapy was associated with longer OS. The authors concluded that RT and paclitaxel-platinum chemotherapy should be attempted whenever feasible for patients with UPSC.

Our results compare very favorably with results of a recent phase II trial that evaluated RT "sandwiched" between paclitaxel-platinum chemotherapy in patients with stage I-IV UPSC [23]. That study found 3-year disease-free survival and OS rates of $69 \%$ and $75 \%$, respectively, for patients with stage I-II disease and 54\% and 52\%, respectively, for patients with stage III-IV disease. 
The choice of chemotherapy regimen remains controversial for women with UPSC. The use of paclitaxel with or without carboplatin or cisplatin for UPSC was first described by Zanotti et al. [11], who reported objective responses in 7 of 11 women treated for recurrent disease (64\%) and 8 of 9 women (89\%) treated for residual disease after initial surgery; 8 of 15 women (53\%) with stage III or IV UPSC were alive at 10 to 62 months of follow-up. Another study of 74 patients with surgically staged stage I UPSC found a $3 \%$ recurrence rate among patients treated with platinum-based chemotherapy compared to $47 \%$ in patients who did not receive chemotherapy [23]. This translated into a disease-free survival advantage ( $\mathrm{p}$ $<0.01$ ) and an OS advantage ( $\mathrm{p}<0.05$ ) for patients treated with a platinum-based regimen. However, other studies of platinum-based therapy for UPSC have shown much lower response rates (i.e., about 25\%) [8-10], which led investigators at MD Anderson to undertake a trial of paclitaxel alone. These investigators found an overall response rate of $77 \%$ in 20 women with advanced-stage or recurrent UPSC [12]. On the basis of those results, we chose to test paclitaxel-only chemotherapy in the present study, and we achieved excellent results with minimal chemotherapy-related toxic effects.

The rate of grade 3 or more severe late bowel toxic effects in our study was $13 \%$ and included 3 bowel obstructions. The rate of grade 3 or more severe diarrhea during pelvic RT was $43 \%$. By comparison, Martinez et al. reported a $14 \%$ rate of chronic gastrointestinal toxic effects in patients treated with WAPI [7]. Our paclitaxel dose of $50 \mathrm{mg} / \mathrm{m}^{2}$ weekly, which we chose on the basis of published findings indicating that this dose is associated with minimal and manageable toxic effects [16-18], may have been too high for our relatively elderly patients (mean age, 63 years); a dose of $40 \mathrm{mg} / \mathrm{m}^{2}$ weekly might have been better tolerated. Another potential means of reducing bowel toxic effects, the use of IMRT, was not allowed in our study. IMRT has shown evidence of reducing both acute and long-term bowel toxic effects by reducing the dose to the small and large bowel [24-29].

Recently, data have been published that indicate that vaginal brachytherapy may provide the same benefit as pelvic RT with less toxicity. A large randomized study showed that vaginal brachytherapy was comparable to pelvic radiation therapy in regards to LC and OS in patients with intermediate risk endometrial carcinoma [30]. This study did not include patients with UPSC. There are very few studies available on the use of vaginal brachytherapy in patients with UPSC. One very small study showed a $13 \%$ relapse rate with use of vaginal brachytherapy alone for patients with stage I/II UPSC [31]. The Gynecologic Oncology Group has activated a trial (GOG 0249) that may help address the use of vaginal brachytherapy and chemotherapy in patients with stage I-II UPSC. However, until we have the results of this trial, there is very little evidence that vaginal cuff brachytherapy is sufficient treatment for patients with all stage of UPSC. It may be sufficient for patients with surgical stage IA disease limited to the polyp, but there are very few data at present to recommend this treatment.

In conclusion, we found that concurrent paclitaxel and pelvic RT followed by 4 courses of systemic paclitaxel produced favorable results in patients with surgically staged UPSC, results that were similar to other published reports. Although this treatment was tolerated fairly well, tolerance may potentially be improved by dose reduction of concomitant paclitaxel to $40 \mathrm{mg} / \mathrm{m}^{2}$ weekly and use of IMRT for pelvic RT. The excellent LC and OS 
rates achieved in this study lead us to recommend that this treatment be considered for patients with this aggressive form of endometrial carcinoma who are believed to have a high risk of pelvic recurrence, such as patients with stage IB, IC, II, or IIIC (pelvic nodes only) disease. However, patients with stage IA and very minimally invasive stage IB disease may be better treated with vaginal cuff brachytherapy plus chemotherapy.

\section{References}

1. Creasman WT, et al. Prognosis of papillary serous, clear cell, and grade 3 stage I carcinoma of the endometrium. Gynecol Oncol. 2004; 95(3):593-6. [PubMed: 15581969]

2. Huh WK, et al. Uterine papillary serous carcinoma: comparisons of outcomes in surgical Stage I patients with and without adjuvant therapy. Gynecol Oncol. 2003; 91(3):470-5. [PubMed: 14675664]

3. Kelly MG, et al. Improved survival in surgical stage I patients with uterine papillary serous carcinoma (UPSC) treated with adjuvant platinum-based chemotherapy. Gynecol Oncol. 2005; 98(3):353-9. [PubMed: 16005947]

4. Kwon J, Ackerman I, Franssen E. The role of abdominal-pelvic radiotherapy in the management of uterine papillary serous carcinoma. Int J Radiat Oncol Biol Phys. 2004; 59(5):1439-45. [PubMed: 15275730]

5. Slomovitz BM, et al. Uterine papillary serous carcinoma (UPSC): a single institution review of 129 cases. Gynecol Oncol. 2003; 91(3):463-9. [PubMed: 14675663]

6. Turner BC, et al. Effective treatment of stage I uterine papillary serous carcinoma with high doserate vaginal apex radiation (192Ir) and chemotherapy. Int J Radiat Oncol Biol Phys. 1998; 40(1): 77-84. [PubMed: 9422561]

7. Martinez AA, et al. Improved outcome at 10 years for serous-papillary/clear cell or high-risk endometrial cancer patients treated by adjuvant high-dose whole abdomino-pelvic irradiation. Gynecol Oncol. 2003; 90(3):537-46. [PubMed: 13678721]

8. Chambers JT, et al. Uterine papillary serous carcinoma treated with intraperitoneal cisplatin and intravenous doxorubicin and cyclophosphamide. Gynecol Oncol. 1996; 60(3):438-42. [PubMed: 8774654]

9. Levenback C, et al. Uterine papillary serous carcinoma (UPSC) treated with cisplatin, doxorubicin, and cyclophosphamide (PAC). Gynecol Oncol. 1992; 46(3):317-21. [PubMed: 1526508]

10. Price FV, et al. Intravenous cisplatin, doxorubicin, and cyclophosphamide in the treatment of uterine papillary serous carcinoma (UPSC). Gynecol Oncol. 1993; 51(3):383-9. [PubMed: 8112650]

11. Zanotti KM, et al. The use of paclitaxel and platinum-based chemotherapy in uterine papillary serous carcinoma. Gynecol Oncol. 1999; 74(2):272-7. [PubMed: 10419744]

12. Ramondetta L, et al. Treatment of uterine papillary serous carcinoma with paclitaxel. Gynecol Oncol. 2001; 82(1):156-61. [PubMed: 11426978]

13. Liebmann $\mathbf{J}$, et al. In vitro studies of Taxol as a radiation sensitizer in human tumor cells. J Natl Cancer Inst. 1994; 86(6):441-6. [PubMed: 7907149]

14. Tishler RB, et al. Taxol: a novel radiation sensitizer. Int J Radiat Oncol Biol Phys. 1992; 22(3): 613-7. [PubMed: 1346533]

15. Wahl AF, et al. Loss of normal p53 function confers sensitization to Taxol by increasing G2/M arrest and apoptosis. Nat Med. 1996; 2(1):72-9. [PubMed: 8564846]

16. Chen MD, et al. Phase I trial of taxol as a radiation sensitizer with cisplatin in advanced cervical cancer. Gynecol Oncol. 1997; 67(2):131-6. [PubMed: 9367695]

17. Glantz MJ, et al. Weekly paclitaxel with and without concurrent radiation therapy: toxicity, pharmacokinetics, and response. Semin Oncol. 1996; 23(6 Suppl 16):128-35. [PubMed: 9007140]

18. Safran $\mathrm{H}$, et al. Paclitaxel and concurrent radiation for locally advanced pancreatic and gastric cancer: a phase I study. J Clin Oncol. 1997; 15(3):901-7. [PubMed: 9060526] 
19. Shepherd JH. Revised FIGO staging for gynaecological cancer. Br J Obstet Gynaecol. 1989; 96(8): 889-92. [PubMed: 2775686]

20. Fader AN, et al. Platinum/taxane-based chemotherapy with or without radiation therapy favorably impacts survival outcomes in stage I uterine papillary serous carcinoma. Cancer. 2009; 115(10): 2119-27. [PubMed: 19306417]

21. Goldberg $\mathrm{H}$, et al. Outcome after combined modality treatment for uterine papillary serous carcinoma: a study by the Rare Cancer Network (RCN). Gynecol Oncol. 2008; 108(2):298-305. [PubMed: 18096209]

22. Viswanathan AN, et al. The importance of chemotherapy and radiation in uterine papillary serous carcinoma. Gynecol Oncol. 123(3):542-7. [PubMed: 21963091]

23. Kelly MG, et al. Patients with uterine papillary serous cancers may benefit from adjuvant platinum-based chemoradiation. Gynecol Oncol. 2004; 95(3):469-73. [PubMed: 15581948]

24. Alongi F, et al. IMRT significantly reduces acute toxicity of whole-pelvis irradiation in patients treated with post-operative adjuvant or salvage radiotherapy after radical prostatectomy. Radiother Oncol. 2009; 93(2):207-12. [PubMed: 19766338]

25. Beriwal S, et al. Clinical outcome with adjuvant treatment of endometrial carcinoma using intensity-modulated radiation therapy. Gynecol Oncol. 2006; 102(2):195-9. [PubMed: 16647748]

26. Chen MF, et al. Adjuvant concurrent chemoradiotherapy with intensity-modulated pelvic radiotherapy after surgery for high-risk, early stage cervical cancer patients. Cancer J. 2008; 14(3): 200-6. [PubMed: 18536561]

27. Mundt AJ, et al. Intensity-modulated whole pelvic radiotherapy in women with gynecologic malignancies. Int J Radiat Oncol Biol Phys. 2002; 52(5):1330-7. [PubMed: 11955746]

28. Mundt AJ, Mell LK, Roeske JC. Preliminary analysis of chronic gastrointestinal toxicity in gynecology patients treated with intensity-modulated whole pelvic radiation therapy. Int J Radiat Oncol Biol Phys. 2003; 56(5):1354-60. [PubMed: 12873680]

29. Roeske JC, et al. A dosimetric analysis of acute gastrointestinal toxicity in women receiving intensity-modulated whole-pelvic radiation therapy. Radiother Oncol. 2003; 69(2):201-7. [PubMed: 14643959]

30. Nout RA, et al. Vaginal brachytherapy versus pelvic external beam radiotherapy for patients with endometrial cancer of high-intermediate risk (PORTEC-2): an open-label, non-inferiority, randomised trial. Lancet. 375(9717):816-23. [PubMed: 20206777]

31. DuBeshter B, et al. High-dose rate brachytherapy for Stage I/II papillary serous or clear cell endometrial cancer. Gynecol Oncol. 2004; 94(2):383-6. [PubMed: 15297176] 


\section{Highlights}

- Trial looking at concurrent chemoradiation followed by adjuvant chemotherapy in patient with stage I-IIIA papillary serous carcinoma.

- This regime was well tolerated.

- Better OS and PFS in patients with stage I and IIIA compared to historical control. 


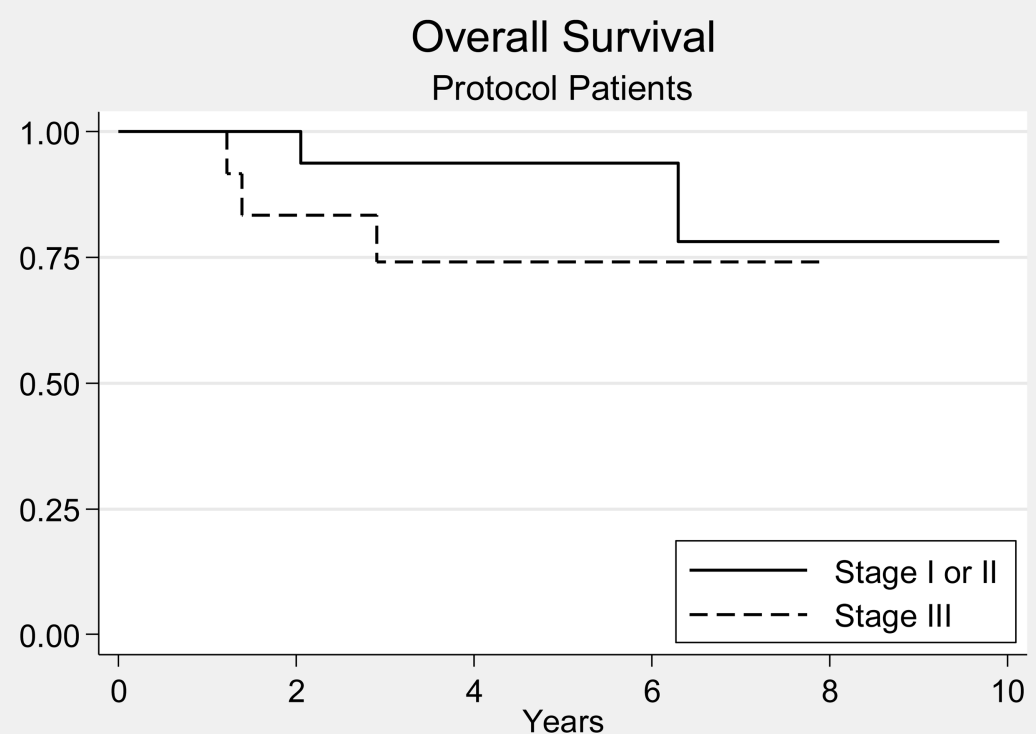

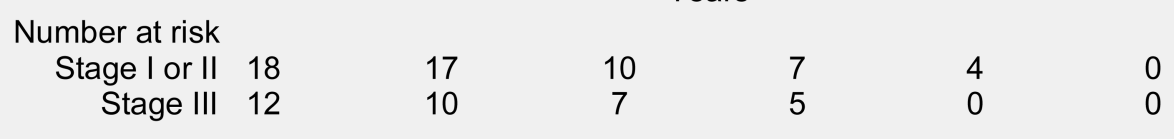

Figure 1.

Overall survival rates for all patients on the protocol. There was no significant difference in overall survival between the patients with stage I-II disease and those with stage IIIA disease.[5] 


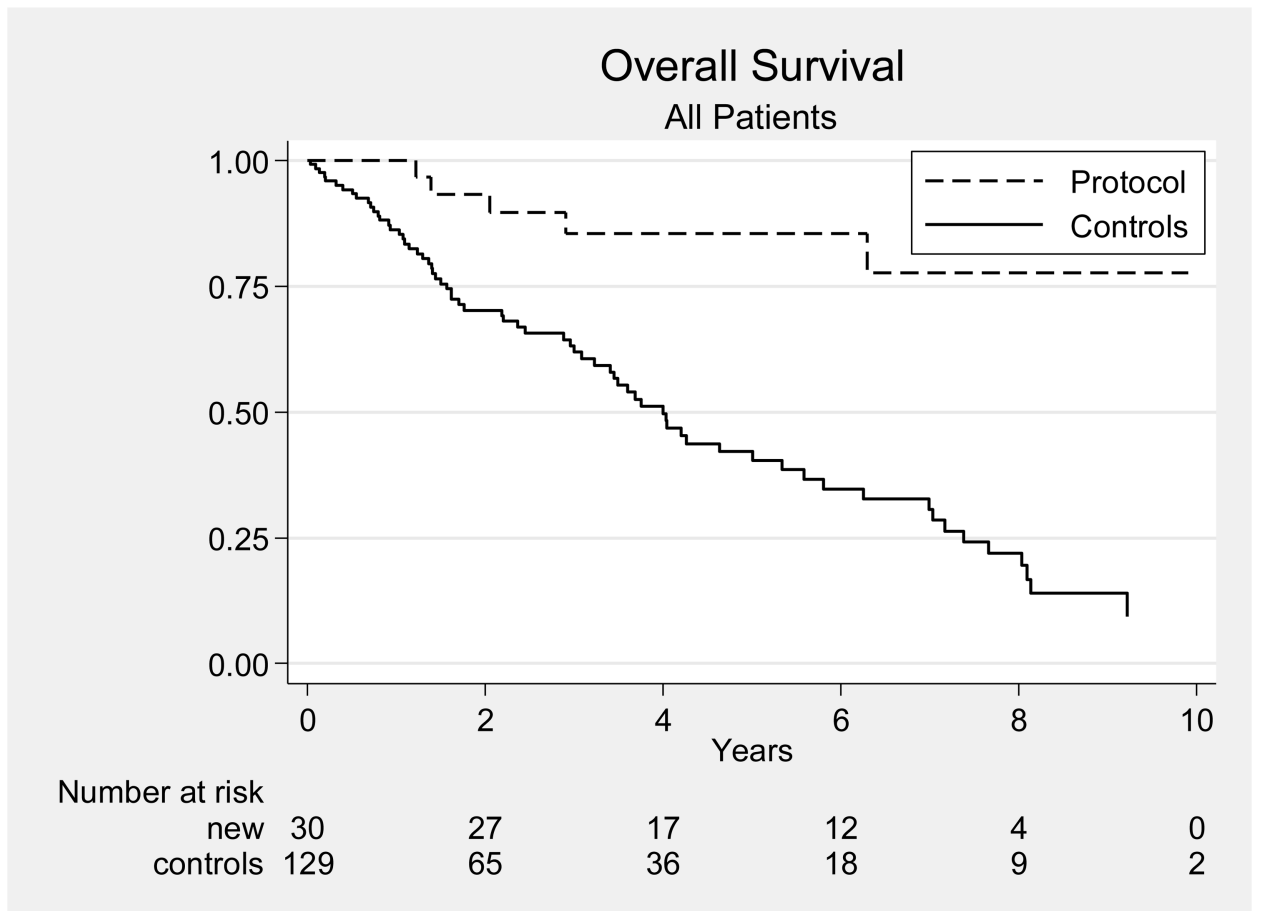

Figure 2.

Overall survival rates for all patients on the protocol ("Protocol") and patients in a historical series ("Controls"). The protocol patients are the new patients, and the historical series are called controls. The x-axis represents years.[5] 


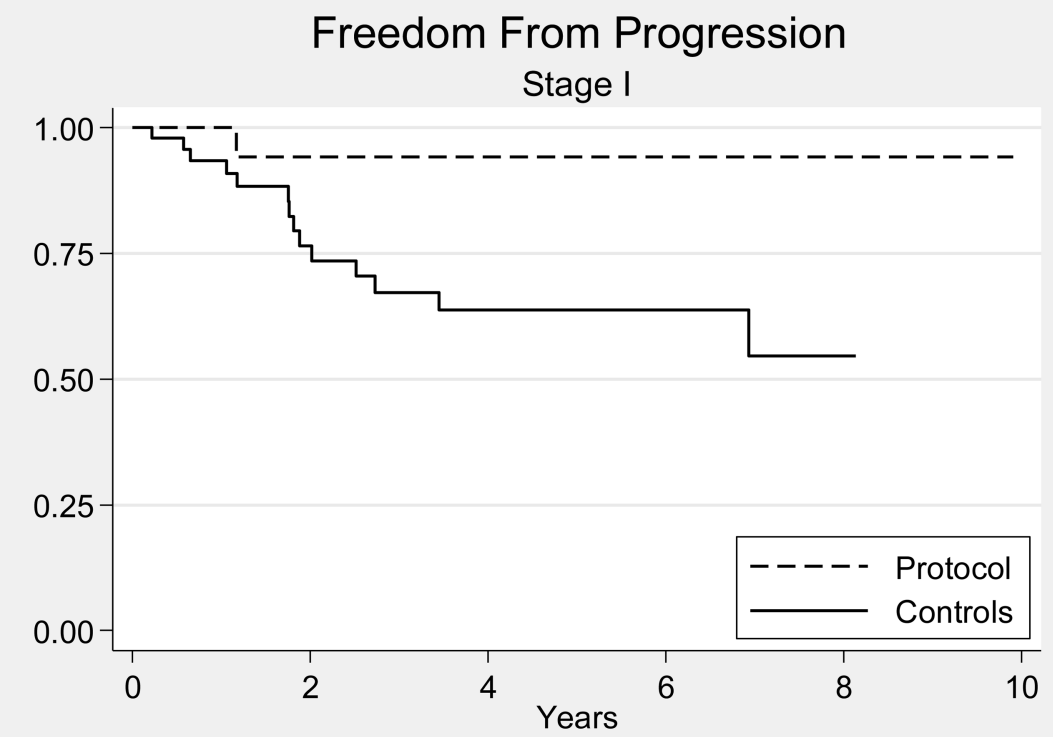

Number at risk

new 17

controls 51

15

9

7

2

0

26

15

10

Figure 3.

Progression-free survival rates for patients with stage I disease in the current study and in the historical series.[5] 

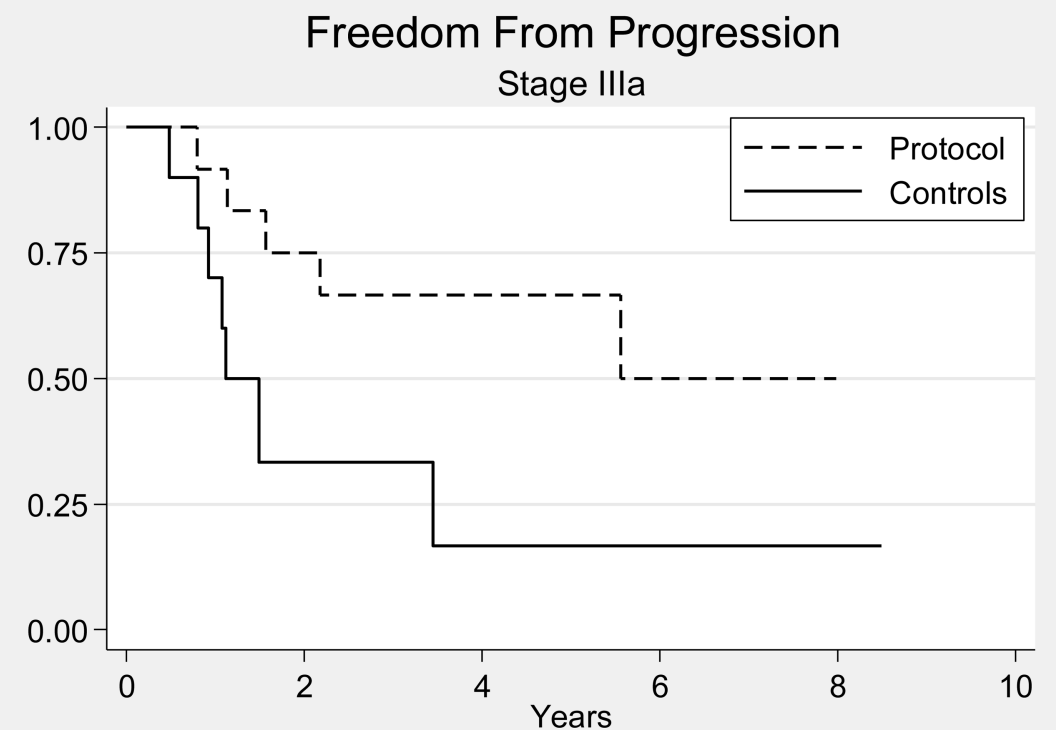

Number at risk

new 12

controls 14

$9 \quad 6$

6

3

2

1

1

0

0

Figure 4.

Progression-free survival rates for patients with stage IIIA disease in the current study and in the historical series.[5] 


\section{Table 1}

Patient characteristics.

\begin{tabular}{lcc}
\hline Characteristic & N & \% \\
\hline Race/ethnicity & & \\
Asian & 1 & 3 \\
Black & 3 & 10 \\
Hispanic & 3 & 10 \\
White & 23 & 77 \\
Disease stage & & \\
IA & 3 & 10 \\
IB & 11 & 37 \\
IC & 3 & 10 \\
IIB & 1 & 3 \\
IIIA & 12 & 40 \\
Cytologic washings & & \\
Positive & 11 & 37 \\
Negative & 19 & 63 \\
Lymphovascular space invasion \\
Yes & 12 & 40 \\
No & 18 & 60 \\
Ovary/fallopian tube involvement \\
Positive & 5 & 17 \\
Negative & 25 & 83 \\
\hline & &
\end{tabular}

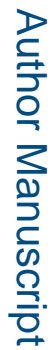

ace/ethnicity

Disease stage 

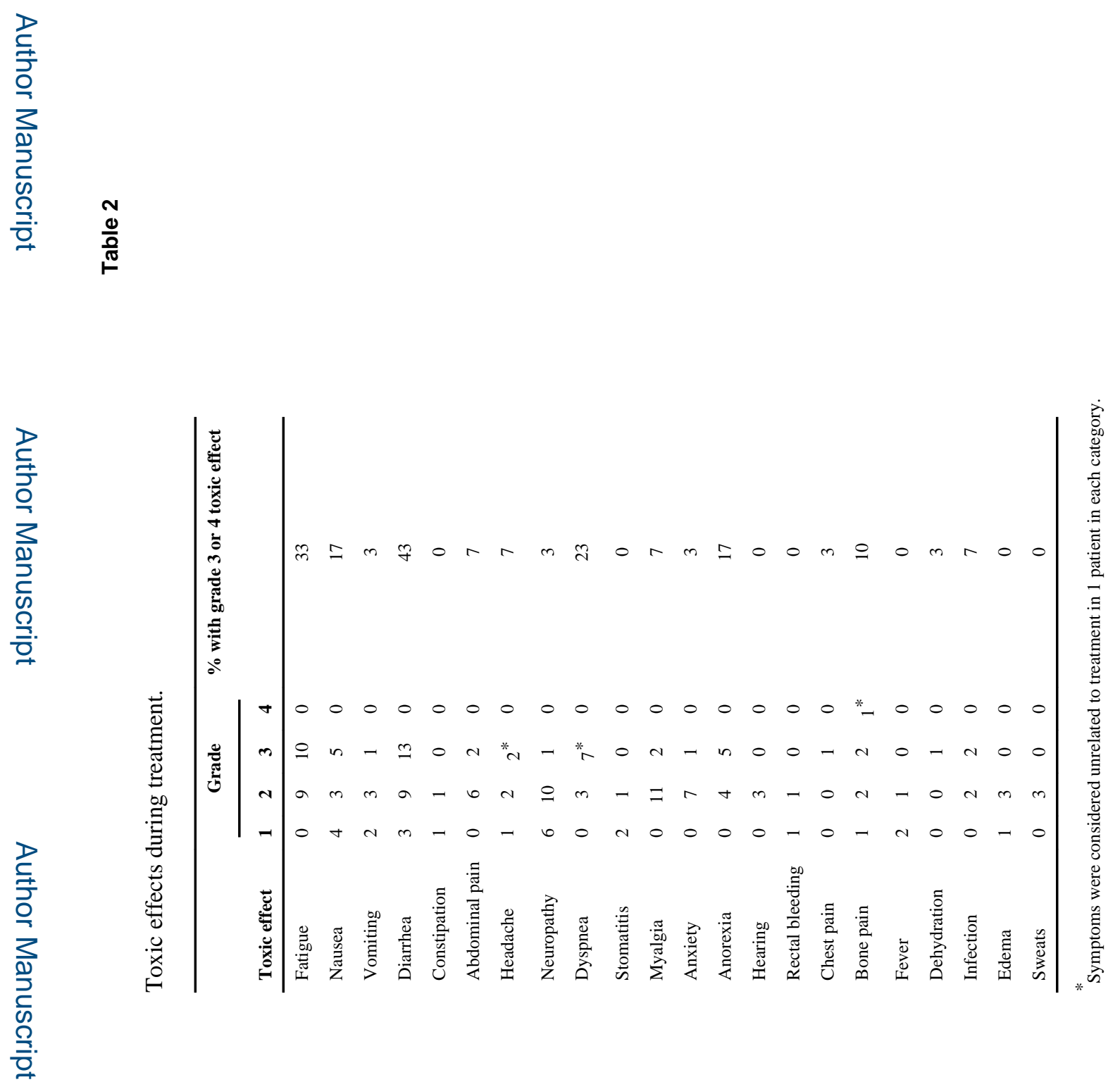

Gynecol Oncol. Author manuscript; available in PMC 2016 March 29. 


\section{Table 3}

Hematologic toxic effects during treatment.

\begin{tabular}{lllll}
\hline & \multicolumn{4}{c}{ Grade } \\
\cline { 2 - 5 } Toxicity & $\mathbf{1}$ & $\mathbf{2}$ & $\mathbf{3}$ & $\mathbf{4}$ \\
\hline Hemoglobin & 11 & 15 & 0 & 0 \\
Platelets & 4 & 0 & 3 & 0 \\
Total leukocyte counts & 7 & 18 & 3 & 0 \\
Absolute neutrophil counts & 2 & 9 & 4 & 3 \\
\hline
\end{tabular}

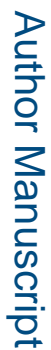

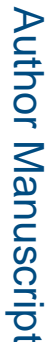

